\title{
New Pacemaker Implantation in Patients undergoing Transcatheter Valve Replacement: a systematic review and meta-analysis
}

\author{
Hilaryano Ferreira ( $\square$ hilaryano.ferreira@hff.min-saude.pt) \\ Hospital Prof. Doutor Fernando Fonseca, Amadora, Portugal \\ Humberto Morais \\ Hospital Militar Principal/Instituto Superior, Luanda, Angola \\ Ana Rita Ferreira \\ Hospital Prof. Doutor Fernando Fonseca, Amadora, Portugal \\ Francisco Madeira \\ Hospital Prof. Doutor Fernando Fonseca, Amadora, Portugal \\ Carlos Morais \\ Hospital Prof. Doutor Fernando Fonseca, Amadora, Portugal
}

\section{Systematic Review}

Keywords: Pacing, Cardiac, TAVI, TAVR, electrophysiology, meta-analysis

Posted Date: August 5th, 2021

DOI: https://doi.org/10.21203/rs.3.rs-784339/v1

License: (c) (i) This work is licensed under a Creative Commons Attribution 4.0 International License. Read Full License 


\section{Abstract}

Background

Permanent pacemaker (PPM) implantation remain a common finding after Transcatheter aortic valve replacement (TAVR). Overall rate of PPM implantation after TAVR varies and is related to various factors and is highly variable. The purpose of this review is to evaluate the incidence of new permanent pacemaker implant at 1 year irrespective of valve brand manufacturer, vascular access used, deployment technique/mechanism. Secondary outcomes included all-cause mortality, 30-day, 1-year mortality, and cardiovascular mortality.

Methods

We performed a systematic search for studies that reported the incidence of PPM implantation after TAVR. Data on study, patient, and procedural characteristics were abstracted. Risk ratios (RRs); odds ratio (OR) and 95\% confidence intervals were calculated by use of random effects models.

Results

14 studies were included, totalling 25,967 TAVR patients, mean age $80.15 \pm 6.91$ years, $52.9 \%$ being male, $26.3 \%$ of which required PPM implantation 1 -year after intervention ( $p=0.00001 ;$ RR 115.16).

Conclusions

Various factors impact the risk for pacemaker implantation after TAVR and one quoter of the patients undergo new permanent pacemaker implant at 1-year post undergoing TAVR.

\section{Introduction}

Aortic valve replacement (AVR) is the mainstay of treatment of symptomatic severe aortic stenosis (AS). Transcatheter aortic valve implantation (TAVl; or transcatheter aortic valve replacement [TAVR]) is now more a safe and feasible alternative to surgical aortic valve replacement (SAVR), especially in high surgical risk patients. ${ }^{1,2}$ Among procedural complications including arrhythmias (eg, conduction abnormalities and atrial fibrillation) may arise. Risk factors such as atrioventricular block, pre-existing right bundle branch block, new left bundle branch block and use of a CoreValve (versus SAPIEN valve) have been associated need for new permanent pacemaker (PPM) implantation. ${ }^{3,4}$ Post-TAVR overall new PPM implantation rate varies and is related to pre-procedural and intraprocedural factors. Some studies reported rates of new PPM or intracardiac defibrillator implant of about 8-17 percent. ${ }^{5-7}$ Other studies report incidence as high as $21-42.5$ percent. 8,9

Current data regarding the impact of TAVR on conduction system, new PPM requirement on mortality is still conflicting.

In this meta-analysis we tend to evaluate the incidence of new permanent pacemaker implant at 1 year irrespective of valve brand manufacturer, vascular access used, deployment technique/mechanism (auto-expandable vs mechanical). Secondary outcomes included all-cause mortality, 30-day, 1-year mortality, and cardiovascular mortality.

\section{Methods}

\section{Overview}

We did a systematic review and meta-analysis of studies on prevalence and outcomes of arrhythmic mitral annular disjunction. The review is reported according to PRISMA guidelines.

\section{Search strategy, selection criteria, and data extraction}

We electronically searched the PUBMED databases with no starting date through June, 2021. The search included MeSH term ['TAVR' and 'pacemaker'] according to the Preferred Reporting Items for Systematic Reviews and Meta-Analyses statement, PRISMA. No language restrictions were applied. Additional search strategy included limiting the search applying filters: 'Clinical Trials' and published in the last 5 years.

Abstracts and potentially relevant full texts were reviewed independently by two authors (HF and HM) with any conflicts resolved by consensus. Case-control studies, cohort studies, and brief reports were not eligible for inclusion (prespecified by search strategy). Case reports, studies without comparison groups, narrative or systematic literature reviews, preprint papers, and studies reporting on overlapping populations were excluded. The following data were extracted: author's name, publication date, study design, study population, follow-up period.

A total of fourteen (14) studies were included in the meta-analysis. ${ }^{10-23}$

Primary outcomes included (rate of new pacemaker implantation in patients undergoing transcatheter aortic valve replacement at 1 year). We intended to evaluate the primary outcome irrespective of valve brand manufacturer, vascular access used, deployment technique/mechanism (auto-expandable vs mechanical). Secondary outcomes included (all-cause mortality at 1-year, 30-day mortality, and cardiovascular mortality). We planned to record any additional patients' outcomes identified. Where papers described service configuration or resource-use changes without clinical outcomes, we excluded them from the analysis. 
Quality appraisal

The quality appraisal was established according to Le Floch and colleagues' criteria, by two independent assessors (HF and HM) ${ }^{24}$ This tool appraises the quality of the study based on the following questions: Did this article give an answer to the research question? Did the article focus clearly on the research question? Was the methodology appropriate? Do you believe the results? (Can it be due to chance, bias or confounding?). To be included, the article had to score "yes" on every question.

Statistical analysis

Quantitative meta-analysis was done for an outcome when more than one study presented relevant data. We excluded individual outcomes from studies reporting no adverse outcomes in one or both groups, and studies not satisfying the normality assumption for continuous variables.

A random-effects estimate of the pooled odds of each outcome was generated with use of the Mantel-Haenszel method. Between-study heterogeneity was explored using the $\mathrm{I}^{2}$ statistic, with substantial heterogeneity defined as an $\mathrm{I}^{2}$ value greater than $50 \%$. We reported $\mathrm{p}$ values and the amount of accounted heterogeneity for each covariate. Potential publication bias was assessed with Egger's test and funnel plots for visual inspection when sufficient studies $(n>10)$ were available. Statistical analyses were performed using the Revman software package (Review Manager, Version 5.4. Copenhagen, The Nordic Cochrane Centre, the Cochrane Collaboration).

\section{Results}

Characterization of the study population:

Twenty-five thousand nine hundred and sixty-seven patients totalled from the fourteen studies included. The characteristics of the patients are shown in Table 1. The average age was $80.15 \pm 6.91$ years, $52.9 \%$ being male.

The prevalence of reported hypertension and coronary artery disease was high $76.6 \%$ and $59.7 \%$, respectively; diabetes was reported in $30.2 \%$. Of the studies that reposted the prevalence of heart failure was $49.3 \%$. Pre-procedural risk was assessed by the logistic EuroSCORE or the STS-PROM score in the majority of included studies, although in 5 studies, did not report on the risk score.

Primary outcome:

The overall prevalence of new permanent pacemaker implantation occurred in $6844(26.3 \%)$ of the 25,967 patients $(p=.00001)(\mathrm{RR} 115.16[95 \% \mathrm{Cl} 29.80$ 415.10]; $\left.P^{2}=69 \%\right)$. Figure 1

Secondary outcomes:

All-cause mortality was reported in thirteen studies ${ }^{10-21,23}$ totalling 25875 patients. All-cause mortality occurred in 1613 (6.2\%) patients (OR 51.84 [95\% Cl 11.35-236.68]). Table 230 -day mortality rate reported by 8 studies ${ }^{10-15,19,21}$ occurring in 104 (2.4\%) of the 4287 patients (OR 30.47 [95\%Cl 9.58-96.87]).

One-year mortality rate reported by 8 studies ${ }^{10-15,219,21}$ occurred in 398 (9.2\%) of the 4287 patients (OR 94.44 [95\%Cl 30.06-296.71]).

Cardiovascular mortality reported in 9 studies ${ }^{10-13,15,18,19,20,21}$ occurring in 806 (3.2\%) of the 25004 patients (OR 94.57 [95\%Cl $\left.\left.26.46-337.99\right]\right)$.

\section{Discussion}

Post-TAVR conduction disturbances such as new onset left bundle brunch block (LBBB) are still the one of the main setbacks of the procedure. It may occur within the first 24 hours post-procedure, even though most events acutely during valve expansion, new-onset LBBB may occur before valve implantation, during guidewire insertion and balloon predilation. ${ }^{25,26}$

The results of the present studies showed that about one quarter (26.3\%) implanted permanent pacemaker (PPM) at 1-year. Previous studies report a 1-year implant rate between $5-20 \% .{ }^{27,28}$ Recent published data from the MARE study reports an annual rate of PPM implantation of $7.3 \% .{ }^{29}$

New LBBB may occur beyond 6 months and at 1 -year in about $57 \%$ of the patients, and in up to $2.9 \%$ of patients beyond 1 -year. ${ }^{30,31}$ In this study we did not assess the clinical predictors of as it was not in our scope and there have been reported by other studies. Diabetes mellitus, previous coronary artery bypass graft, female sex, the amount of calcification of the aortic valve, and pre-existing conduction abnormalities (e.g. prolonged QRS duration) have been identified as risk factors new onset LBBB. ${ }^{32,33,34}$

In the present analysis we taught to investigate the incidence of post-TAVR PPM implant, irrespective of valve brand, deployment mechanism and/or vascular access because previous studies reports on incidence are highly variable. Previous studies assessing the rate of PPM post-TAVR using first generation valves range from $2-51 \%{ }^{35}$

There have been some contradictory data regarding mortality in patients with TAVR and new PPM, suggesting a possible correlation and increased mortality. ${ }^{36,37}$ In our meta-analysis we did not assess the correlation between PPM on mortality as we did not have access to individual patient data and subgroups. As so, we evaluated overall mortality rates. However, a meta-analysis showed that PPI post-TAVR was not associated with any increased risk of all- 
cause mortality at 1 year $(\mathrm{RR}, 1.03 ; 95 \% \mathrm{Cl}, 0.9-1.18)$, furthermore there was a trend towards a protective effect on cardiac death was observed (RR, 0.78 ; $95 \%$ $\mathrm{Cl}, 0.60-1.03){ }^{38}$

\section{Study Limitations}

The current study had following limitations: 1) there were several clinical variables, but we did not aim to systematically examine them. 2) Follow-up data as well as clinical outcomes related to PPM were not reported in most of the studies. Thus, we could not address the clinical long-term effectiveness of PPM implantation in these patients. 3) A clear indication/diagnosis leading to pacemaker implantation was not possible assess in most of the studies.

\section{Conclusions}

Various factors impact the risk for pacemaker implantation after TAVR and although there's

Still conflicting data regarding the incidence, this study showed that at least one quoter of the patients undergo new permanent pacemaker implant at 1-year post undergoing TAVR. Adverse effects of new pacemaker on morbidity and mortality after TAVR, as well as long term mortality must be evaluated in by further research to improve risk-stratification and better identify predictors of poor outcome.

\section{Declarations}

\section{Authors contributions:}

Study design/data analysis (HF), re-checking of data collected (HM/ARF), Drafting article (HF), Critical revision of article article approval of article (FM/CM).

\section{Conflicts of interest}

The authors have no conflicts of interest to declare.

\section{Funding}

No funding or grants was involved in the production of this manuscript.

\section{References}

1. Baumgartner H, Falk V, Bax JJ, De Bonis M, Hamm C, Holm PJ, lung B, et al; ESC Scientific Document Group. 2017 ESC/EACTS Guidelines for the management of valvular heart disease. Eur Heart J. 2017 Sep 21;38(36):2739-2791. doi: 10.1093/eurheartj/ehx391. PMID: 28886619.

2. Otto CM, Nishimura RA, Bonow RO, Carabello BA, Erwin JP 3rd, Gentile F, Jneid H, Krieger EV, Mack M, McLeod C, O'Gara PT, Rigolin VH, Sundt TM 3rd, Thompson A, Toly C. 2020 ACC/AHA Guideline for the Management of Patients With Valvular Heart Disease: A Report of the American College of Cardiology/American Heart Association Joint Committee on Clinical Practice Guidelines. Circulation. 2021 Feb 2;143(5):e72-e227. doi: 10.1161/CIR.0000000000000923. Epub 2020 Dec 17. Erratum in: Circulation. 2021 Feb 2;143(5):e229. PMID: 33332150.

3. Koos R, Mahnken AH, Aktug O, Dohmen G, Autschbach R, Marx N, Hoffmann R. Electrocardiographic and imaging predictors for permanent pacemaker requirement after transcatheter aortic valve implantation. J Heart Valve Dis. 2011 Jan;20(1):83-90. PMID: 21404902.

4. Holmes DR Jr, Mack MJ, Kaul S, Agnihotri A, Alexander KP, Bailey SR, Calhoon JH, et al. 2012 ACCF/AATS/SCAI/STS expert consensus document on transcatheter aortic valve replacement. J Am Coll Cardiol. 2012 Mar 27;59(13):1200-54. doi: 10.1016/j.jacc.2012.01.001. Epub 2012 Jan 31. PMID: 22300974.

5. Holmes DR Jr, Nishimura RA, Grover FL, Brindis RG, Carroll JD, Edwards FH, Peterson ED, et al; STS/ACC TVT Registry. Annual Outcomes With Transcatheter Valve Therapy: From the STS/ACC TVT Registry. J Am Coll Cardiol. 2015 Dec 29;66(25):2813-2823. doi: 10.1016/j.jacc.2015.10.021. Epub 2015 Nov 30. PMID: 26652232.

6. Thourani VH, Kodali S, Makkar RR, Herrmann HC, Williams M, Babaliaros V, Smalling R, et al. Transcatheter aortic valve replacement versus surgical valve replacement in intermediate-risk patients: a propensity score analysis. Lancet. 2016 May 28;387(10034):2218-25. doi: 10.1016/S0140-6736(16)30073-3. Epub 2016 Apr 3. PMID: 27053442

7. Schofer J, Colombo A, Klugmann S, Fajadet J, DeMarco F, Tchétché D, Maisano F, et al. Prospective multicenter evaluation of the direct flow medical transcatheter aortic valve. J Am Coll Cardiol. 2014 Mar 4;63(8):763-8. doi: 10.1016/j.jacc.2013.10.013. Epub 2013 Nov 6. PMID: 24211506.

8. Popma JJ, Adams DH, Reardon MJ, Yakubov SJ, Kleiman NS, Heimansohn D, Hermiller J Jr, et al.; CoreValve United States Clinical Investigators. Transcatheter aortic valve replacement using a self-expanding bioprosthesis in patients with severe aortic stenosis at extreme risk for surgery. $\mathrm{J}$ Am Coll Cardiol. 2014 May 20;63(19):1972-81. doi: 10.1016/j.jacc.2014.02.556. Epub 2014 Mar 19. PMID: 24657695.

9. Zahn R, Gerckens U, Grube E, Linke A, Sievert H, Eggebrecht H, Hambrecht R, et al; German Transcatheter Aortic Valve Interventions-Registry Investigators. Transcatheter aortic valve implantation: first results from a multi-centre real-world registry. Eur Heart J. 2011 Jan;32(2):198-204. doi: 10.1093/eurheartj/ehq339. Epub 2010 Sep 23. PMID: 20864486.

10. Popma JJ, Deeb GM, Yakubov SJ, Mumtaz M, Gada H, O'Hair D, Bajwa T, et al.; Evolut Low Risk Trial Investigators. Transcatheter Aortic-Valve Replacement with a Self-Expanding Valve in Low-Risk Patients. N Engl J Med. 2019 May 2;380(18):1706-1715. doi: 10.1056/NEJMoa1816885. Epub 2019 Mar 16. PMID: 30883053. 
11. Reardon MJ, Van Mieghem NM, Popma JJ, Kleiman NS, Søndergaard L, Mumtaz M, Adams DH, et al.; SURTAVI Investigators. Surgical or Transcatheter Aortic-Valve Replacement in Intermediate-Risk Patients. N Engl J Med. 2017 Apr 6;376(14):1321-1331. doi: 10.1056/NEJMoa1700456. Epub 2017 Mar 17. PMID: 28304219.

12. Gleason TG, Reardon MJ, Popma JJ, Deeb GM, Yakubov SJ, Lee JS, Kleiman NS, et al.; CoreValve U.S. Pivotal High Risk Trial Clinical Investigators. 5-Year Outcomes of Self-Expanding Transcatheter Versus Surgical Aortic Valve Replacement in High-Risk Patients. J Am Coll Cardiol. 2018 Dec 4;72(22):26872696. doi: 10.1016/j.jacc.2018.08.2146. Epub 2018 Sep 21. PMID: 30249462.

13. Waksman R, Rogers T, Torguson R, Gordon P, Ehsan A, Wilson SR, Goncalves J, et al. Transcatheter Aortic Valve Replacement in Low-Risk Patients With Symptomatic Severe Aortic Stenosis. J Am Coll Cardiol. 2018 Oct 30;72(18):2095-2105. doi: 10.1016/j.jacc.2018.08.1033. Epub 2018 Aug 28. PMID: 30170075.

14. Deharo P, Bisson A, Herbert J, Lacour T, Saint Etienne C, Grammatico-Guillon L, Porto A, et al. Impact of Sapien 3 Balloon-Expandable Versus Evolut R SelfExpandable Transcatheter Aortic Valve Implantation in Patients With Aortic Stenosis: Data From a Nationwide Analysis. Circulation. 2020 Jan 28;141(4):260-268. doi: 10.1161/CIRCULATIONAHA.119.043971. Epub 2019 Nov 16. PMID: 31736332.

15. Feldman TE, Reardon MJ, Rajagopal V, Makkar RR, Bajwa TK, Kleiman NS, Linke A, et al. Effect of Mechanically Expanded vs Self-Expanding Transcatheter Aortic Valve Replacement on Mortality and Major Adverse Clinical Events in High-Risk Patients With Aortic Stenosis: The REPRISE III Randomized Clinical Trial. JAMA. 2018 Jan 2;319(1):27-37. doi: 10.1001/jama.2017.19132. PMID: 29297076; PMCID: PMC5833545.

16. Akodad M, Roubille F, Marin G, Lattuca B, Macia JC, Delseny D, Gandet T, et al. Myocardial Injury After Balloon Predilatation Versus Direct Transcatheter Aortic Valve Replacement: Insights From the DIRECTAVI Trial. J Am Heart Assoc. 2020 Dec 15;9(24):e018405. doi: 10.1161/JAHA.120.018405. Epub 2020 Dec 10. PMID: 33297821; PMCID: PMC7955361.

17. Yamashita K, Fujita T, Fukushima S, Shimahara Y, Kume Y, Matsumoto Y, Kawamoto N, et al. Transcatheter Aortic Valve Replacement for Severe Aortic Stenosis Complicated by Sigmoid Septum. Circ J. 2018 Nov 24;82(12):3090-3099. doi: 10.1253/circj.CJ-18-0264. Epub 2018 Oct 5. PMID: 30298851.

18. Lei WH, Liao YB, Wang ZJ, Ou YW, Tsauo JY, Li YJ, Xiong TY, et al. Transcatheter Aortic Valve Replacement in Patients with Aortic Stenosis Having Coronary Cusp Fusion versus Mixed Cusp Fusion Nonraphe Bicuspid Aortic Valve. J Interv Cardiol. 2019 Nov 3;2019:7348964. doi: 10.1155/2019/7348964. PMID: 31777470; PMCID: PMC6874966.

19. Gleason TG, Schindler JT, Hagberg RC, Deeb GM, Adams DH, Conte JV, Zorn GL 3rd, et al. Subclavian/Axillary Access for Self-Expanding Transcatheter Aortic Valve Replacement Renders Equivalent Outcomes as Transfemoral. Ann Thorac Surg. 2018 Feb;105(2):477-483. doi: 10.1016/j.athoracsur.2017.07.017. Epub 2017 Nov 1. PMID: 29100645.

20. Toutouzas K, Benetos G, Voudris V, Drakopoulou M, Stathogiannis K, Latsios G, Synetos A, et al. Pre-Dilatation Versus No Pre-Dilatation for Implantation of a Self-Expanding Valve in All Comers Undergoing TAVR: The DIRECT Trial. JACC Cardiovasc Interv. 2019 Apr 22;12(8):767-777. doi: 10.1016/j.jcin.2019.02.005. Epub 2019 Mar 27. PMID: 30928442.

21. Søndergaard L, Rodés-Cabau J, Hans-Peter Linke A, Fichtlscherer S, Schäfer U, Kuck KH, Kempfert J, et al. Transcatheter Aortic Valve Replacement With a Repositionable Self-Expanding Prosthesis: The PORTICO-I Trial 1-Year Outcomes. J Am Coll Cardiol. 2018 Dec 11;72(23 Pt A):2859-2867. doi: 10.1016/j.jacc.2018.09.014. Epub 2018 Sep 24. PMID: 30261238.

22. Sager SJ, Damluji AA, Cohen JA, Shah S, O'Neill BP, Alfonso CE, Martinez CA, et al. Transient and persistent conduction abnormalities following transcatheter aortic valve replacement with the Edwards-Sapien prosthesis: a comparison between antegrade vs. retrograde approaches. $J$ Interv Card Electrophysiol. 2016 Nov;47(2):143-151. doi: 10.1007/s10840-016-0145-2. Epub 2016 May 28. PMID: 27236653.

23. Seeger J, Gonska B, Rottbauer W, Wöhrle J. New generation devices for transfemoral transcatheter aortic valve replacement are superior compared with last generation devices with respect to VARC-2 outcome. Cardiovasc Interv Ther. 2018 Jul;33(3):247-255. doi: 10.1007/s12928-017-0477-6. Epub 2017 Jun 22. PMID: 28643214.

24. Le Floch B, Bastiaens H, Le Reste JY, Lingner H, Hoffman RD, Czachowski S, Assenova R, et al. Which positive factors determine the GP satisfaction in clinical practice? A systematic literature review. BMC Fam Pract. 2016 Sep 13;17(1):133. doi: 10.1186/s12875-016-0524-x. PMID: 27619913; PMCID: PMC5020554.

25. Massoullié G, Bordachar P, Ellenbogen KA, Souteyrand G, Jean F, Combaret N, Vorilhon C, et al. New-Onset Left Bundle Branch Block Induced by Transcutaneous Aortic Valve Implantation. Am J Cardiol. 2016 Mar 1;117(5):867-73. doi: 10.1016/j.amjcard.2015.12.009. Epub 2015 Dec 13. PMID: 26742470 .

26. Nuis RJ, Van Mieghem NM, Schultz CJ, Tzikas A, Van der Boon RM, Maugenest AM, Cheng J, et al. Timing and potential mechanisms of new conduction abnormalities during the implantation of the Medtronic CoreValve System in patients with aortic stenosis. Eur Heart J. 2011 Aug;32(16):2067-74. doi: 10.1093/eurheartj/ehr110. Epub 2011 May 28. PMID: 21622979.

27. Muntané-Carol G, Guimaraes L, Ferreira-Neto AN, Wintzer-Wehekind J, Junquera L, Del Val D, Faroux L, et al. How does new-onset left bundle branch block affect the outcomes of transcatheter aortic valve repair? Expert Rev Med Devices. 2019 Jul;16(7):589-602. doi: 10.1080/17434440.2019.1624161. Epub 2019 Jun 7. PMID: 31172837.

28. R Alves Pinto, T Proenca, M Martins Carvalho, S Torres, PD Grilo, CX Resende, AF Amador, et al, Conduction disturbances after TAVR - a 1-year follow-up, EP Europace, Volume 23, Issue Supplement_3, May 2021, euab116.110, https://doi.org/10.1093/europace/euab116.110

29. Muntané-Carol G, Urena M, Nombela-Franco L, Amat-Santos I, Kleiman N, Munoz-Garcia A, Atienza F, et al. Arrhythmic burden in patients with new-onset persistent left bundle branch block after transcatheter aortic valve replacement: 2-year results of the MARE study. Europace. 2021 Feb 5;23(2):254-263. doi: 10.1093/europace/euaa213. PMID: 33083813.

30. Nazif TM, Williams MR, Hahn RT, Kapadia S, Babaliaros V, Rodés-Cabau J, Szeto WY, et al. Clinical implications of new-onset left bundle branch block after transcatheter aortic valve replacement: analysis of the PARTNER experience. Eur Heart J. 2014 Jun 21;35(24):1599-607. doi: 
10.1093/eurheartj/eht376. Epub 2013 Oct 30. PMID: 24179072.

31. Houthuizen P, van der Boon RM, Urena M, Van Mieghem N, Brueren GB, Poels TT, Van Garsse LA, et al. Occurrence, fate and consequences of ventricular conduction abnormalities after transcatheter aortic valve implantation. Eurolntervention. 2014 Feb;9(10):1142-50. doi: 10.4244/EIJV9I10A194. PMID: 24273252.

32. Houthuizen P, Van Garsse LA, Poels TT, de Jaegere P, van der Boon RM, Swinkels BM, Ten Berg JM, et al. Left bundle-branch block induced by transcatheter aortic valve implantation increases risk of death. Circulation. 2012 Aug 7;126(6):720-8. doi: 10.1161/CIRCULATIONAHA.112.101055. Epub 2012 Jul 12. PMID: 22791865.

33. Schymik G, Tzamalis P, Bramlage P, Heimeshoff M, Würth A, Wondraschek R, Gonska BD, et al. Clinical impact of a new left bundle branch block following TAVI implantation: 1-year results of the TAVIK cohort. Clin Res Cardiol. 2015 Apr;104(4):351-62. doi: 10.1007/s00392-014-0791-2. Epub 2014 Nov 12. PMID: 25388650.

34. Hein-Rothweiler R, Jochheim D, Rizas K, Egger A, Theiss H, Bauer A, Massberg S, et al. Aortic annulus to left coronary distance as a predictor for persistent left bundle branch block after TAVI. Catheter Cardiovasc Interv. 2017 Mar 1;89(4):E162-E168. doi: 10.1002/ccd.26503. Epub 2016 Apr 1. PMID: 27038099.

35. Siontis GC, Jüni P, Pilgrim T, Stortecky S, Büllesfeld L, Meier B, Wenaweser P, et al. Predictors of permanent pacemaker implantation in patients with severe aortic stenosis undergoing TAVR: a meta-analysis. J Am Coll Cardiol. 2014 Jul 15;64(2):129-40. doi: 10.1016/j.jacc.2014.04.033. PMID: 25011716.

36. L Fauchier, A Bodin, A Bisson, J Herbert, T Lacour, C Saint Etienne, J.M Clerc, et al. Outcomes of permanent pacemaker implantation following transcatheter aortic valve replacement, European Heart Journal, Volume 41, Issue Supplement_2, November 2020, ehaa946.0719,

https://doi.org/10.1093/ehjci/ehaa946.0719

37. Fadahunsi OO, Olowoyeye A, Ukaigwe A, Li Z, Vora AN, Vemulapalli S, Elgin E, et al. Incidence, Predictors, and Outcomes of Permanent Pacemaker Implantation Following Transcatheter Aortic Valve Replacement: Analysis From the U.S. Society of Thoracic Surgeons/American College of Cardiology TVT Registry. JACC Cardiovasc Interv. 2016 Nov 14;9(21):2189-2199. doi: 10.1016/j.jcin.2016.07.026. PMID: 27832844.

38. Regueiro A, Abdul-Jawad Altisent O, Del Trigo M, Campelo-Parada F, Puri R, Urena M, Philippon F, et al. Impact of New-Onset Left Bundle Branch Block and Periprocedural Permanent Pacemaker Implantation on Clinical Outcomes in Patients Undergoing Transcatheter Aortic Valve Replacement: A Systematic Review and Meta-Analysis. Circ Cardiovasc Interv. 2016 May;9(5):e003635. doi: 10.1161/CIRCINTERVENTIONS.115.003635. PMID: 27169577.

\section{Tables}

Table.1 Study and Patients characteristics at baseline

\begin{tabular}{|c|c|c|c|c|c|c|c|c|c|c|c|c|c|c|}
\hline $\begin{array}{l}\text { JE PATIENT } \\
\text {;TERISTICS }\end{array}$ & $\begin{array}{c}\text { Popma et } \\
\text { al }\end{array}$ & $\begin{array}{l}\text { Reardon } \\
\text { et.al }\end{array}$ & $\begin{array}{l}\text { Gleason } \\
2018 \text { et.al }\end{array}$ & $\begin{array}{c}\text { Waksman } \\
\text { et.al }\end{array}$ & Deharo et.al & $\begin{array}{l}\text { Feldman } \\
\text { et.al }\end{array}$ & $\begin{array}{l}\text { Akodad } \\
\text { et.al }\end{array}$ & $\begin{array}{c}\text { Yamashita } \\
\text { et.al }\end{array}$ & Lei et.al & $\begin{array}{c}\text { Gleason } 2017 \\
\text { et.al }\end{array}$ & $\begin{array}{c}\text { Toutouzas } \\
\text { et.al }\end{array}$ & $\begin{array}{l}\text { Sendergaard } \\
\text { et.al }\end{array}$ & Sager et.al & $\begin{array}{l}\text { Seeger } \\
\text { et.al }\end{array}$ \\
\hline $\begin{array}{l}\text { Study type } \\
\text { ctive/prospective) }\end{array}$ & Prospective & Prospective & Prospective & Prospective & Retrospective & Prospective & Prospective & Retrospective & Retrospective & Retrospective & Prospective & Prospective & Retrospective & Prospective \\
\hline Age, mean/SD & $74.0 \pm 5.9$ & $79.9 \pm 6.2$ & $83.2 \pm 7.1$ & $73.6 \pm 6.1$ & $82.55 \pm 6.77$ & $82.85 \pm 7.3$ & $83 \pm 9$ & $83.9 \pm 5.5$ & $71.9 \pm 5.8$ & $80.5 \pm 8.9$ & $81.7 \pm 7.17$ & $82.4 \pm 5.9$ & $86 \pm 9$ & $80.7 \pm 6.1$ \\
\hline כx (male), n (\%) & 65.1 & 498 (57.6) & $207(52.9)$ & $123(61.5)$ & $\begin{array}{l}10883 \\
(53.4)\end{array}$ & 449 (49.2) & $\begin{array}{c}129 \\
(61.1 \%)\end{array}$ & $70(37)$ & $32(45.1)$ & $248(61.3)$ & $90(52.6)$ & $322(34.3)$ & $46(50)$ & $176(44)$ \\
\hline rtension, $\mathrm{n}(\%)$ & $614(84.8)$ & $801(92.7)$ & $372(95.1)$ & $171(85.5)$ & $\begin{array}{l}16553 \\
(81.2)\end{array}$ & NR & $137(64.9)$ & NA & $32(45.1)$ & $376(93.0)$ & NR & 759 (80.7) & $81(88)$ & NR \\
\hline betes, n (\%) & $228(31.4)$ & $295(34.1)$ & $136(34.8)$ & $61(30.5)$ & $6044(29.6)$ & $286(31.3)$ & $79(37.4)$ & $59(31.2)$ & $14(19.7)$ & $174(43.0)$ & $56(32.7)$ & $278(29.6)$ & $34(37)$ & $121(30.2)$ \\
\hline pidemia, n (\%) & NR & NR & NR & NR & $9579(47)$ & NR & NR & $120(63.4)$ & NR & NR & NR & NR & NR & NR \\
\hline эco use, n (\%) & NR & NR & NR & NR & $1688(8.3)$ & NR & NR & NR & NR & $248(61.3)$ & NR & NR & $57(62)$ & NR \\
\hline : Failure, n (\%) & NR & $824(95.4)$ & 373 (95.4) & NR & $11020(54)$ & NR & NR & NR & NR & $393(97.2)$ & NR & $125(13.3)$ & $71(77.2)$ & NR \\
\hline $\begin{array}{l}\text { Artery Disease, } \mathrm{n} \\
(\%)\end{array}$ & $120(16.4)$ & $541(62.6)$ & $295(75.4)$ & $44(23)$ & $\begin{array}{l}12593 \\
(61.7)\end{array}$ & $657(72)$ & $104(49.2)$ & NR & $25(35.2 \%)$ & $334(82.6)$ & $78(45.6)$ & $473(50.3)$ & NR & $256(64)$ \\
\hline TS PROM $^{\dagger}$ & $1.9 \pm 0.7$ & $4.4 \pm 1.5$ & $7.3 \pm 3.0$ & $1.8 \pm 0.5$ & NR & $6.7 \pm 4.0$ & NR & $7.45 \pm 4.75$ & $7.0 \pm 3.6$ & $9.76 \pm 5.7$ & NR & $5.8 \pm 4.8$ & NR & $7.4 \pm 5.2$ \\
\hline uroSCORE ${ }^{\pi}$ & NR & $11.9 \pm 7.6$ & $\begin{array}{c}17.7 \pm \\
13.0\end{array}$ & NR & NR & $6.5 \pm 5.5$ & $2.6 \pm 2$ & NR & NR & $20 \pm 14.65$ & $24.3 \pm 10.68$ & $15.7 \pm 11.3$ & NR & NR \\
\hline SYNTAX $^{\ddagger}$ & $1.9 \pm 3.7$ & NR & NR & NR & NR & NR & NR & NR & NR & NR & NR & NR & NR & NR \\
\hline \multicolumn{15}{|l|}{$\begin{array}{l}\text { liography } \\
\S \\
\text { ss }\end{array}$} \\
\hline $\begin{array}{l}\text {-valve gradient, } \\
\text { ImHg (SD) }\end{array}$ & $47.0 \pm 12.1$ & $\begin{array}{c}47.2 \pm \\
14.3\end{array}$ & NR & NR & NA & $\begin{array}{c}44.24 \\
\pm 12.83 \\
\end{array}$ & $27.75 \pm 40$ & $47.05 \pm 14.65$ & NR & NR & $48.31 \pm 14.88$ & $49.7 \pm 15.3$ & NR & $36.0 \pm 16.5$ \\
\hline $\begin{array}{l}\text {-valve area, } \mathrm{cm} 2 \\
\text { (SD) }\end{array}$ & $0.8 \pm 0.2$ & $0.8 \pm 0.2$ & NR & NR & NA & $\begin{array}{c}0.69 \pm \\
0.19\end{array}$ & $0.75 \pm 0.9$ & $0.70 \pm 0.18$ & NR & NR & $0.67 \pm 0.15$ & $0.7 \pm 0.3$ & NR & $0.79 \pm 0.29$ \\
\hline $\begin{array}{l}\text { ntricular ejection } \\
\text { stion, \% (SD) }\end{array}$ & $61.7 \pm 7.9$ & NR & NR & $63.5 \pm 7.5$ & NA & NR & $60 \pm 10$ & $58.5 \pm 8.5$ & NR & NR & $50.46 \pm 9.79$ & $57.4 \pm 11.6$ & NR & $54 \pm 14.4$ \\
\hline
\end{tabular}

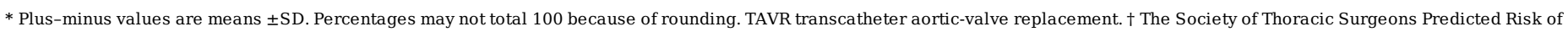

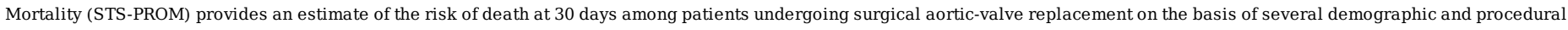

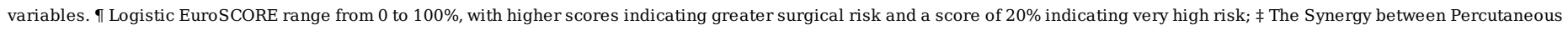

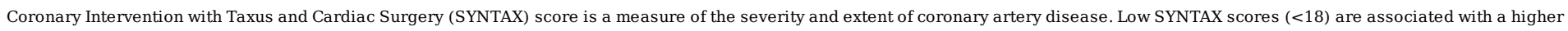
success rate with PCI, scores between 18 and 27 with an intermediate success rate, and scores higher than 27 with a low success rate. $\S$ These data were reported by the studies. 
Table.2 Secondary Outcomes

\begin{tabular}{|c|c|c|c|}
\hline Outcome & N studies reporting & $\mathbf{n / N}$ & Odds Ratio (M-H, Random, 95\% CI) \\
\hline All-cause mortality & 13 & $1613 / 25875$ & $51.84[11.35,236.68]$ \\
\hline 30-day mortality & 8 & $104 / 4287$ & $30.47[9.58,96.87]$ \\
\hline 1-year mortality & 8 & $398 / 4287$ & $94.44[30.06,296.71]$ \\
\hline Cardiovascular mortality & 9 & $806 / 25004$ & $94.57[26.46,337.99]$ \\
\hline
\end{tabular}

\section{Figures}

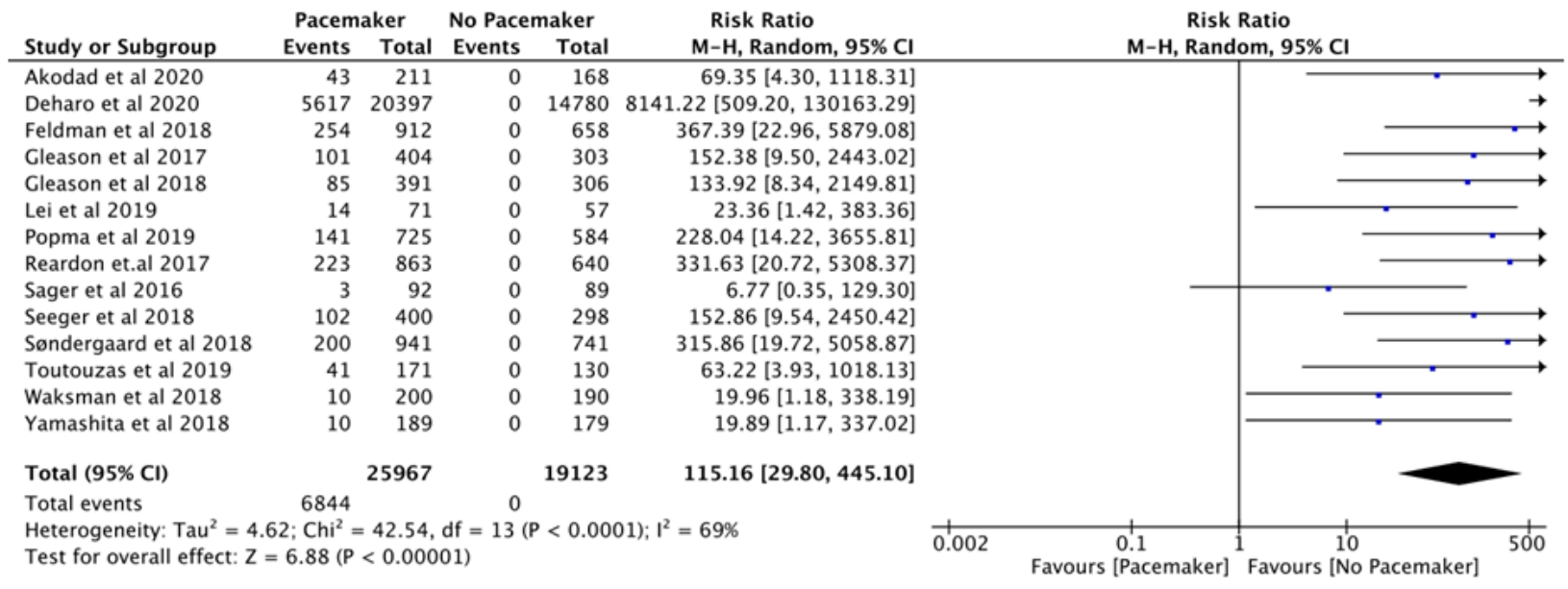

\section{Figure 1}

Primary Outcome

\section{Supplementary Files}

This is a list of supplementary files associated with this preprint. Click to download.

- PRISMAFlowDiagram.png

- GraphicalAbstract.png 